\title{
Diagnostic laparoscopy in chronic abdominal diseases
}

\author{
Mishra N. ${ }^{1}$, Sharma S. ${ }^{2}$ \\ ${ }^{1}$ Dr Navneet Mishra, Assistant Professor, ${ }^{2}$ Dr Shrikant Sharma, Associate Professor; both authors are affiliated with \\ Department of Surgery, L.N. Medical College \& J.K. Hospital, Kolar Road, Bhopal, MP, India.
}

Address for Correspondence: Dr Shrikant Sharma, Associate Professor, Department of Surgery, L.N. Medical College \& J.K. Hospital, Kolar Road, Bhopal. E-mail: drshrikantsharma@gmail.com

\begin{abstract}
Objectives: To establish an early diagnosis of the diseases where clinical findings are unreliable, with additional information about extent, nature, morbid changes and operability of the diseases andto establish that laparoscopy can prevent diagnostic laparotomy. Methodology: The present study was carried out in 32 patients admitted in surgical, medical, gynaecological and cancer wards. The patients were followed and planned for furthermanagement according to the laparoscopic findingseither conservatively or exploratory. The patients having inoperable malignancy were referred to cancer hospital for further medical management and patients with koch's abdomen were started antitubercular treatment. Results: Laproscopy was helpful in confirming the clinical diagnosis in $46.875 \%$ of cases, itsolved the diagnostic dilemma in $34.375 \%$ of cases and in $9.375 \%$ the clinical impression was foundwrong which was corrected by laparoscopy. In $9.375 \%$ of cases diagnostic laparoscopy was done with the intention of looking secondary deposits, if present, to stage the malignancy. As compared to clinical diagnosis laparoscopic diagnosis was proved wrong in 3.125\% cases, later on during laprotomy. Conclusion: Laparoscopy is a safe, simple, accurate and useful diagnostic modality in the general surgical practice. It is cost effective and can be used in critically ill patients. Thus laparoscopy has a significant diagnostic and therapeutic role in patients with chronic abdominal pain.
\end{abstract}

Key words: Laparoscopy, Abdominal pain, Laprotomy

\section{Introduction}

Abdomen is a "Magic-Box" consisting of a variety of structurally and functionally different organs, protected by the abdominal wall. A lot of acute and chronic abdominal disease are one of the important cause of morbidity and mortality even in this era ofmedical science. Despite the presence of a number of sophisticated diagnostic tools some of the abdominal diseaseremain obscure owing to their confusing clinical presentation.A surgeon commonly relies upon surgical exploration of the abdomen when there is a doubt about the nature of a patient'sprimary illness because he is usually impatient and may feel that a diagnosisis unsatisfactory without introducinghis hands in the abdominal cavity andpalpate the diseased organ[1].

But with the advanced instrumentation, particularly incorporation of fiber-optic techniques, the laparoscopy has become more effective and determinative in evaluating intra-abdominal disease states. Since it is relatively simple, safe, highly informative and permits

Manuscript Received: $20^{\text {th }}$ September 2017

Reviewed: $30^{\text {th }}$ September 2017

Author Corrected: $9^{\text {th }}$ October 2017

Accepted for Publication: $16^{\text {th }}$ October 2017 definitive diagnosis of many acute and chronic intra abdominal condition by direct visualization of the affected organ, together with the biopsy of the representative area, if necessary, laparoscopic procedures should have wider application in modern practice. It is not only economical and less time consuming thanlaparotomy but also has rendered unnecessary a number of more hazardous diagnostic and therapeutic approaches in common use. It also avoids undue delay in the diagnosis which is of utmost importance in acute surgical patients.

Besides in some patients in whom confirm diagnosis cannot be made or in doubt by clinical findings, biochemical and roentgenological findings, radioactive scans or even sophisticated techniques like USG and CT scan, laparoscopy has a definite role.

Since the value of information obtained is undisputed, the current opinion has veered from the principle of 'open and see' to 'peep and see'. In addition, laparoscopic diagnosis can be followed by subsequent laparotomy as and when needed[2]. 


\section{Original Research Article}

Safe general anaesthesia and modern antibiotics have diminished the hazards of laparoscopy. It is therefore reasonable that a surgeon consider the laparoscopy, as of no added benefit over laparotomy alone. It is true that laparotomy can provide all the information inherent to laparoscopy but laparoscopy can provide the same information at a far less discomfort, operative risk, expense and cosmetic compromise. In some cases, like extensive peritoneal metastasis of malignant diseases, inoperable conditions with liver secondary, staging of lymphoma, in medical conditions where patients may not withstand surgery. In these instances laparoscopy can offer an alternative to laprotomy with equal accuracy and reduced risks. So offer an alternative to laprotomy with equal accuracy and reduced risks. So both the procedures are complimentary and must be used wisely and selectively. "When in doubt, cut it out" is the answer to difficult diagnostic problems, otherwise "peep before leap". Difficult areas like dome of diaghragm, omentum, serosal surface of bowel, parietal peritoneum etc can be evaluated under direct vision, photographed and biopsied. "Laparoscopy is principally valuable for what is definitely seen and not for what is apparently absent"[3].

Prime purpose of performing laparoscopy is direct visualization of pathological organs like liver, gall bladder, peritoneum and other viscera, to detect presence of pathological macroscopic changes, their extent, status and to obtain tissue under direct vision for accurate histological confirmation of the disease. Though the modern diagnostic techniques are extremely sensitive and efficacious but resolving power of laparoscopy is far greater.

In our set up where facilities for sophisticated noninvasive procedures are not easily available, laparoscopy should gain popularity as it neither requires specific prerequisites nor any risky anaesthesia. To sum up, with the advancement of fiber optic imaging, lens system and computerization "Key-Hole" surgery is coming up and laparoscopy as a diagnostic procedure is gaining wider acceptance because of reduced risk and cost. The procedure is definitely having a bright future.

\section{Methodology}

A prospective comparative studyentitled "Diagnostic laparoscopy in chronic abdominal diseases" was carried out in 32 patients admitted in surgical, medical gynaecological and cancer wards of L. N. Medical College and associated J. K. Hospital, Bhopal between July 2012 to Aug. 2013. The study was conducted in J K Hospital \& Research Centre, Bhopal which is a 750 bedded multi disciplinary tertiary and referral hospital in Bhopal.

\section{Inclusion criteria}

- ASA 1 and 2 Patients

- No respiratory or cardiac problems

\section{Exclusion criteria}

- Cardiac problems

- Respiratory diseases

- Extreames of age -old and children

- Any existing significant bleedingdiathesis.

Sample Size- Total of 32 patients.

Approvedproforma were used for collecting demographic data, clinical data, preoperative evaluation, intra-operative observations and post-op findings and complications. Written informed consent was taken from patient relatives for surgical procedure and regarding the potential risks of anesthesia and surgery and also post operative evaluation. All cases were given Inj. Voveron (diclofenac sodium 75 mg) I.M. just before leaving the theatre and the same was repeated 8 hourly for the next $48 \mathrm{hrs}$. Patients details were recorded in prescribed proforma and the following observationsmade.

\section{Results}

In the present series, $59.375 \%$ of patients were male and $40.625 \%$ were female. The maximum number of patients belong to the 51-60 years. age group. Laparoscopy alone was performed in 34.375\% of cases, laparoscopy with biopsy in 50\% of cases, laparoscopy with tapping of ascitic fluid in $6.25 \%$ and laparoscopic guided cholecystocholangiography in $6.25 \%$ of cases. The aspiration of liver abscess was done under laparoscopic visual guidance in $3.125 \%$ of cases.

Out of 32 patients, 19 patients (59.375\%) subsequently underwent exploratory laprotomy either for treatment purpose or for confirmation of laparoscopic diagnosis or for treatment of complication of laparoscopy. Laparoscopy was performed in 18 cases oflump in abdomen, of which 13 cases were subjected to follow up laparotomy. In only one case laparoscopic diagnosis was found wrong. The diagnostic accuracy of this group was found $92.3 \%$. In the miscellaneous group of 4 patients, 3 patients underwent laparoscopy for staging of already proved malignancy, of which one patient of CA Esophagus found to have secondaires in liver and spared from exploration, were planned for curative resection. The one patient with suspected 


\section{Original Research Article}

diagnosis of cirrhosis was confirmed to have mixed nodular variety of Cirrhosis by laparoscopic biopsy. The diagnostic accuracy found in this group was $100 \%$ in this present study.

The overall diagnostic accuracy of laparoscopy as compared to clinical and radiological examination found in this series was $94.737 \%$. The incidence of minor complications found in this study was $28.125 \%$ and the incidence of major complication was $3.125 \%$

Table No 1: Distribution of cases according to age and sex.

\begin{tabular}{|c|c|c|c|c|c|c|c|}
\hline S. No. & Age Groups & No of Patients & \% & Male & \% & Female & \% \\
\hline 1 & $11-20$ & 1 & $3.125 \%$ & 1 & $3.125 \%$ & - & - \\
\hline 2 & $21-30$ & 5 & $15.625 \%$ & 3 & $9.375 \%$ & 5 & $6.25 \%$ \\
\hline 3 & $31-40$ & 6 & $18.75 \%$ & 3 & $9.375 \%$ & 3 & $9.375 \%$ \\
\hline 4 & $41-50$ & 6 & $18.75 \%$ & 5 & $15.625 \%$ & 1 & $3.125 \%$ \\
\hline 5 & $51-60$ & 7 & $21.875 \%$ & 4 & $12.5 \%$ & 3 & $9.375 \%$ \\
\hline 6 & $61-70$ & 5 & $15.625 \%$ & 3 & $9.375 \%$ & 2 & $6.25 \%$ \\
\hline 7 & $71-80$ & 2 & 6.25 & - & - & 2 & $6.25 \%$ \\
\hline & Total & $\mathbf{3 2}$ & $\mathbf{1 0 0} \%$ & $\mathbf{1 9}$ & $\mathbf{5 9 . 3 7 5 \%}$ & $\mathbf{1 3}$ & $\mathbf{4 0 . 6 2 5 \%}$ \\
\hline
\end{tabular}

The overall male to female ratio was 3:1 The males were dominating in all age group except in 31-40 years age group where ratio was equal 3:3, and in 71-80 years age group where only 2 female patients were representative. This signifies that chronic abdominal disease were slightly more common in males.

Table No 2: Distributions of case according to indication of laparoscopy.

\begin{tabular}{|c|c|c|c|}
\hline S.N. & Findings & No. of Cases & \% \\
\hline 1 & $\begin{array}{c}\text { Evaluation of abdominallump } \\
\text { (with or without hepatomegaly ) }\end{array}$ & 18 & $56.25 \%$ \\
2 & Hepatomegaly & 5 & $15.625 \%$ \\
3 & Jaundice + lump +hepatomegaly & 11 & $34.375 \%$ \\
4 & Chronic abdominal pain & 19 & $59.375 \%$ \\
5 & Infertility & 2 & $6.25 \%$ \\
6 & Miscellaneous & 4 & $12.5 \%$ \\
\hline
\end{tabular}

The most frequent indication was the evaluation of abdominal lump with or without hepatomegaly and jaundice in $56.25 \%$ of cases followed by evaluation of hepatomegaly. The evaluationof jaundice was the indications in $34.375 \%$ of cases which was associated with lump and/or hepatomegaly. $59.375 \%$ of cases were subjected to laparoscopy for the complaint of chronic abdominal pain in addition to other complaints. $6.25 \%$ of cases were evaluated for cause of infertility.

Table No 3: Procedure performed.

\begin{tabular}{|c|c|c|c|}
\hline S.No. & Procedure & No. of Cases & \% \\
\hline 1 & LaparoscopyAlone & 10 & $31.25 \%$ \\
\hline 2 & Laparoscopy Biopsy & 16 & $50 \%$ \\
\hline 3 & Tapping of Fluid+ Laparoscopy & 2 & $6.25 \%$ \\
\hline 4 & Laparoscopy +Cholecystocholangiography & 2 & $6.25 \%$ \\
\hline 5 & Laparoscopy + Aspiration & 2 & $6.25 \%$ \\
\hline & Total & $\mathbf{3 2}$ & $\mathbf{1 0 0 \%}$ \\
\hline
\end{tabular}

The most commonly carried out procedure was laparoscopic biopsy in 50\% of cases followed by laparoscopy alone in $31.25 \%$ of cases. In $6.25 \%$ of cases laparoscopy was preceded by tapping of ascitic fluid while in same no. of cases transcholecystic cholangiography was done under laparoscopoic visual guidance. These were the cases of cholangiocarcinoma and choledocholithiasis. In two cases (6.25\%) aspiration of Liver abscess was done under visual guidance of laparoscopy. 


\section{Original Research Article}

Clinical diagnosis made on the basis of history and physical examination of the patient was confirmed in more than $64 \%$ of cases when the patients were subjected to diagnosis laparoscopy. The laparoscopy was found more useful in this regard as besides confirmation of clinical diagnosis it also revealed the clinically unidentified secondary deposits in liver \& peritoneum and also the extent of disease. In remaining the clinical diagnosis was modified in view of laparoscopic findings in three cases and uncertain clinical diagnosis was made by laparoscopy in 11 cases. Inthree cases of malignancy, laparoscopy was performed only for staging of malignany. In one case laparoscopic diagnosis made was wrong and found to have Ileocaecal TB lesion but later on to carcinoma caecum on laprotomy.

Table No 4: Accuracy of clinical vs laparoscopic diagnosis.

\begin{tabular}{|c|c|c|c|}
\hline S. No. & Group & No. of cases & $\mathbf{5}$ \\
\hline 1 & Clinical diagnosis was confirmed by laparoscopy & 15 & $46.875 \%$ \\
\hline 2 & Clinical diagnosis was uncertain and made by laparoscopy & 11 & $34.375 \%$ \\
\hline 3 & Clinical diagnosis was wrong and corrected by laparoscopy & 3 & $9.375 \%$ \\
\hline 4 & Laparoscopy was helpful to stage malignancy & 3 & $9.375 \%$ \\
\hline 5 & Misleading laparoscopy & 1 & $3.125 \%$ \\
\hline & Total & $\mathbf{3 2}$ & $\mathbf{1 0 0 \%}$ \\
\hline
\end{tabular}

Confirming the clinical diagnosis in $46.875 \%$ of cases, laparoscopy had solved the diagnostic dielemma in $34.375 \%$ of cases and in $9.375 \%$ the clinical impression was made wrong which was corrected by laparoscopy. In $9.375 \%$ of cases diagnostic laparoscopy was done with the intention of looking secondary deposits, if present, to stage the malignancy. As compared to clinical diagnosis laparoscopic diagnosis was proved wrong in $3.125 \%$ cases, later on laparotomy.

Table No 5: Distribution of cases according to complication of laparoscopy.

\begin{tabular}{|c|c|c|c|}
\hline S. No. & Complication & No. of cases & $\%$ \\
\hline 1 & $\begin{array}{l}\text { Minor } \\
\text {-Surgical emphysema } \\
\text {-local peritonitis } \\
\text {-transient paralytic ileus } \\
\text {-leaking of ascitic fluid }\end{array}$ & $\begin{array}{l}6 \\
3 \\
3 \\
1\end{array}$ & $\begin{array}{l}18.75 \% \\
9.375 \% \\
9.375 \% \\
3.125 \%\end{array}$ \\
\hline 2 & $\begin{array}{l}\text { Major } \\
\text {-Hollow viscera perforation }\end{array}$ & 1 & 3.125 \\
\hline
\end{tabular}

The most common minor complication as listed in the above table was local surgical emphysema around the primary trocar port while insufflating the abdomen with $\mathrm{CO} 2$ and this was resolved within 3-4 days without any consequences. Local peritonitis with mild tenderness and local pain was found in $9.375 \%$ of cases and similar no. of cases had transient paralytic ileus, two of them had surgical emphysema. The ileus was recovered within $48 \mathrm{hrs}$. one patient with moderate malignant ascitis had leakage of ascitic fluid for 1st 2 days which spontaneously ceased. In one patient with gastric lymphoma gastric perforation was the hazard in an attempt to obtain biopsy from the lump. He underwent exploratory laparotomy at an urgent basis but he expired on the same day due to poor general conditions. Thus the major complication encountered in the present series was hollow viscus perforation.

\section{Discussion}

Technological innovation and improved instrumentation have opened up new prospects for minimally invasive surgery, and in the $21^{\text {st }}$ century laparoscopic surgery haschangedthe face of general surgery - both diagnostic and therapeutic. With this aim in mind, the present study entitled, "Diagnostic laparoscopy in chronic abdominal disease" was carried out in 32 cases admitted in the surgical, medical \& gynaecological wards of Medical College \& Associated Hospital, Some patients have more than two symptoms and the laparoscopy was performed for more than one indication. The relevant literature has been collected and reviewed. Easter DW, Cuschieri A et alaudited experience with diagnostic laparoscopy performed over a 30-month period on 131 consecutive patients. Laparoscopy was $100 \%$ accurate in the diagnosis or exclusion of intra-abdominal malignant neoplasms, and future care decisions were affected by this information. Laparoscopy in the evaluation of chronic abdominal pain yielded positive findings in $47 \%$ of cases. The majority of referrals $(73 \%)$ for the investigation of chronic abdominal pain came from the medical services, whereas the majority of referrals $(72 \%)$ for the investigation of cancer 


\section{Original Research Article}

originated from surgeons. Laparoscopy in the setting of acute abdominal pain yielded useful information that affected patient outcomes in $71 \%$ of cases. There were no serious complications in this series. Laparoscopy is a safe and useful diagnostic tool, especially for the diagnosis, staging, or exclusion of cancer.So their findings were similar to our study [4].

Onders RP, Mittendorf EAet al studiedutility of laparoscopy in chronic abdominal pain. Their study was undertaken to assess the utility of performing diagnostic and therapeutic laparoscopy in patients with chronic abdominal pain for longer than 12 weeks. The patients' demographic data, length of time with pain, number of diagnostic studies performed before surgery, intraoperative findings, interventions, pathology, and longterm follow-up were determined.Findings included adhesions in 39 , a hernia in 13 , adhesions from the appendix to adjacent structures in 6, appendiceal pathology in 5, endometriosis in 3, and gallbladder pathology in 2. Ten patients had no obvious pathology. At the time of their initial postoperative visit, $90 \%$ reported their pain to be gone or improved No patient experienced any long-term complications and all reported satisfaction with their procedure[5].

Salky BA, Edye M Bet aldid similar studies andsaw the role of laparoscopy in the diagnosis and treatment of abdominal pain syndromes. Diagnostic laparoscopy plays a significant role in the evaluation of acute and chronic abdominal pain in the era of therapeutic laparoscopic surgery. In thisseries, 387 consecutive patients underwent laparoscopy because of abdominal pain. In a group of 121 patients with acute abdominal pain, a definitive diagnosis was made in 119 cases (98\%). Two patients needed laparotomy to confirm the diagnosis; both had a disease process that did not require laparotomy to treat. A definitive therapeutic laparoscopic procedure was performed in 53 cases $944 \%$ ). In 45 patients (38\%), a diagnosis was made that did not require therapeutic laparoscopy or laparotomy to treat. There was one false negative laparoscopy that required laparotomy to treat 1 month later. So theyconcluded that laparoscopy is an accurate modality for the diagnosis of both acute and chronic abdominal pain syndromes. These data support the use of laparoscopy as the primary invasive intervention in patients with acute and chronic abdominal pain[6].

Nagy AG, James D et al examined the value of laparoscopy as a diagnostic aid. Seventy-seven consecutive patients who underwentprocedure are reported. In 31 patients, laparoscopy was performed for assessment of the cause of acute abdominal pain.
Diagnosis was achieved in 28 patients (90 percent) and laparotomy was avoided in 17 (55 percent). Assessment of chronic abdominal pain in 11 patients yielded a diagnostic accuracy in 9 (82 percent) and laparotomy was avoided in 7 (64 percent). In 11 patients with abdominal trauma, diagnostic accuracy was 91 percent (10 of 11 patients) and laparotomy was not required in 6 (54 percent). In 21 patients with intraabdominal malignancy, 14 (67 percent) were accurately assessed, and in 8 (38 percent) formal exploration was spared. Three patients with obscure causes of ascites and jaundice were all accurately assessed without need for laparotomy. Based on our data, we believe the reports in the literature are reproducible by any abdominal surgeon who uses laparoscopy as a diagnostic aid in their practice [7].

Paajanen $\mathrm{H}$, Julkunen $\mathrm{K}$ et al did a prospective nonrandomized study of 72 patients (60 women and 12 men). One surgeon performed a total of 79 diagnostic laparoscopies including 61 adhesiolysis. The patients' demographic data, operative findings, and long-term postoperative course were carefully recorded. Intraabdominal adhesions were found in 61 patients (85\%) in the laparoscopy, gynecologic disorders in 4, chronic appendicitis in 1, and no abnormality in 6 patients. The abdominal wall pain was a likely reason for pain in 12 patients (17\%). The complication rate was minimal. At 1month control, $38 \%$ of the patients were completely free of pain. In the long-term follow-up, chronic abdominal pain was totally healed in 33 A total of 65 patients $(90 \%)$ reported that the surgery had been beneficial for their intractable pain. So similar to our observations they also concluded that by careful selection, for patients with chronic abdominal pain, laparoscopy alleviates the symptoms in more than $70 \%$ of the patients, and it should be considered if other diagnostics tests are negative. [8].

Kresch AJ, Seifer DB etal did laparoscopy in 100 women with chronic pelvic painin the same location for a minimum of six months. These findings were compared with those of 50 asymptomatic women who underwent laparoscopy for tubal ligation. Overall, $83 \%$ of the group with pelvic pain had abnormal pelvic organs as compared with $29 \%$ of the asymptomatic group. Adhesions were the most common pathology accounting for $38 \%$ and pelvic endometriosis accounted for $32 \%$ of the symptomatic group. Results of this study suggest that pelvic pain reported to be in the same location for a minimum of six months, is usually associated with organic pathology [9].

In a prospective nonrandomized long-term follow-up Paajanen $\mathrm{H}$, Julkunen $\mathrm{K}$ et al observed that Eleven patients with tuberculous peritonitis were treated in a London hospital between 1971 and 1978. All the patients 


\section{Original Research Article}

were immigrants (mean age 30 years). The history of abdominal symptoms was often short and sometimes difficult to distinguish from that of Crohn's disease or neoplasm. The diagnosis was made by laparoscopy and target peritoneal biopsy in eight of these patients. Laparoscopy is a safe and effective method of obtaining an early diagnosis in patients with suspected tuberculous peritonitis. Their study results were in consensus with our study[10].

Minimal access surgery has moved the focus of surgery towards reducing the morbidity of patients while maintaining quality of care. Minimal access surgical techniques are now routine for cholecystectomy, Nissen fundoplication for gastro-oesophageal reflux disease, splenectomy, and adrenalectomy. Use of sentinel node biopsy is minimising the morbidity associated with staging breast cancer. Surgical robotics systems will enable a further revolution in minimally invasive techniques. Future developments are likely to be fuelled by patient demand $[11,12]$.

\section{Conclusion}

Laparoscopy is a safe, simple and useful diagnostic modality in the general surgical practice. It is cost effective and can be used in critically ill patients. In cases of abdominal lump, laparoscopy provides the anatomical location and the morphological characters of the lump together with the extent of the disease. It is useful for evaluation of the cause of jaundice with or without lump and /or hepatomegaly and chronic abdominal painto localize the cause when other investigations fail to find out the cause.

Thus, by this study weare able to do histopathological examination, tapping of ascitis fluid and performance of procedures like percautaneous aspiration of liver abscess under visual guidance, transchoecystic cholangiography etc, which is an added benefit. Thus, laproscopy prevents those laparotomies done only for diagnostic purposes.

Laparoscopy provides an accurate staging of the malignant diseases and its operability and thus prevents many non- therapeutic laparotomies.
Conflict of interest: None declared. Funding: Nil, Permission from IRB: Yes

\section{References}

1. Vecchio R, MacFayden BV, Palazzo F. History of laparoscopic surgery. Panminerva Med. 2000 Mar;42(1): 87-90.

2. Spaner SJ, Warnock GL. A briefhistory of endoscopy, laparoscopy, and laparoscopic surgery. J Laparoendosc Adv Surg Tech A. 1997 Dec;7(6):369-73.

3. Lau WY, Leow CK, Li AK. History of endoscopic and laparoscopic surgery. World J Surg. 1997 May;21 (4): 444-53.

4. Easter DW, Cuschieri A, Nathanson LK, Lavelle-Jones M. The utility of diagnostic laparoscopy for abdominal disorders: audit of 120 patients. Archives of Surgery. 1992 Apr 1;127(4):379-83.

5. Onders RP, Mittendorf EA. Utility of laparoscopy in chronic abdominal pain. Surgery. 2003 Oct;134(4):54952; discussion 552-4.

6. Salky BA, Edye MB. The role of laparoscopy in the diagnosis and treatment of abdominal pain syndromes. Surg Endosc. 1998 Jul;12(7):911-4.

7. Nagy AG, James D. Diagnostic laparoscopy. Am J Surg. 1989 May;157(5):490-3.

8. Paajanen H, Julkunen K, Waris H. Laparoscopy in chronic abdominal pain: a prospective nonrandomized long-term follow-up study. Journal of clinical gastroenterology. 2005 Feb 1;39(2):110-4.

9. Kresch AJ, Seifer DB, Sachs LB, Barrese I. Laparoscopy in 100 women with chronic pelvic pain. Obstet Gynecol. 1984 Nov;64(5):672-4.

10. Wolfe JH, Behn AR, Jackson BT. Tuberculous peritonitis and role of diagnostic laparoscopy. Lancet. 1979 Apr 21;1(8121):852-3.

11. Wilmore DW, Kehlet H. Management of patients in fast track surgery. BMJ. 2001 Feb 24;322(7284):473-6.

12. Darzi A, Mackay S. Recent advances in minimal access surgery. BMJ. 2002 Jan 5;324(7328):31-4.

\section{How to cite this article?}

Mishra N, Sharma S. Diagnostic laparoscopy in chronic abdominal diseases. Surgical Update: Int J surg Orthopedics. 2017;3(4): 114-119.doi:10.17511/ijoso.2017.i04.04. 